\title{
The effect of constraints on bi-objective optimisation of geodetic networks
}

\author{
Mehdi Eshagh • Mohammad Amin Alizadeh-Khameneh
}

Received: 23 October 2014 / Accepted: 4 November 2014 / Published online: 29 November 2014

(C) Akadémiai Kiadó 2014

\begin{abstract}
One of the problems in the single-objective optimisation models (SOOMs) for optimising geodetic networks is the contradiction of the controlling constraints, which may lead to their violation or infeasibility in the optimisation process. One way to solve this problem is to use a bi-objective optimisation model (BOOM) instead of SOOMs. In this paper, we will use the BOOM of precision and reliability and investigate the influence of the controlling constraints in a two-dimensional simulated network. Our studies show that the unconstrained BOOM is a good model, which almost fulfils our precision and reliability demands of the network. This model is also economical as more observables are removed from the plan whilst adding the controlling constraints leads to including more observables, which have no significant role.
\end{abstract}

Keywords Removing observable · Optimality · First- and second-order designs · Two-dimensional geodetic network

\section{Introduction}

The geodetic networks are designed for different purposes, but the main important application of such networks is to monitor the deformation of the man-made structures or the natural features of the Earth. Amongst numerous studies about the design of such networks, we mention the work done by Kuang (1991), Blewitt (2000) and Gerasimenko et al. (2000) who studied the design problem of a monitoring network according to the geophysical parameters and fault-mechanics and Yetki et al. (2008) used the particle swarm optimisation algorithm

\footnotetext{
M. Eshagh $(\bowtie)$

Department of Engineering Science, University West, Trollhättan, Sweden e-mail: eshagh@kth.se; mehdi.eshagh@hv.se
}

M. A. Alizadeh-Khameneh

Division of Geodesy and Geoinformatics, Royal Institute of Technology (KTH), Stockholm, Sweden e-mail:maak3@kth.se 
for a similar purpose. Doma (2013) also implemented this technique to optimise a global positioning system (GPS) network.

Generally, an optimal geodetic network has high precision and reliability, which is designed according to economic considerations. The first step of the geodetic network design is so-called the zero-order design (ZOD) in which the best datum of the network is defined. Different criteria exist for the ZOD. Teunissen (1985) presented the ZOD according to the theory of generalised matrix inverses and its relations with datum and rank deficiency of the design matrix. Kuang (1996) presented different criteria for the ZOD, and Eshagh (2005) suggested the minimum norm and trace of the co-factor matrix as the best criteria for datum definition. There are two well-known ways to find the best configuration of networks, i.e., the first-order design (FOD). One can use either the trial and error or the analytical approaches. In the former, the objective function (OF) is computed with a proposed solution for the problem. If the suggested solution does not satisfy the OF, the solution is changed and the OF is computed again. This process is repeated until the requirement is satisfied. The analytical approaches take advantage of a mathematical algorithm and design the network in such a way that the quality requirement of the network is satisfied. A pioneer in using optimisation theory for the FOD purpose was Koch $(1982,1985)$ who used the quadratic programming theory (Bazaraa and Shetty 1979) to optimise the configuration of a network. Kuang (1991, 1996) and Amiri-Seemkooei (1998) studied this issue further and considered different types of optimisation methods. Berne and Baselga (2004) used the simulated annealing method for the FOD and Amiri-Seemkooei (2008) presented an analytical approach for the same purpose.

Grafarend (1975) and Schmitt (1980, 1985) presented different approaches for the secondorder design (SOD) where the observables' weights and types are determined. Xu (1989) developed a multi-objective optimisation model (MOOM) for the SOD purpose and Kuang (1993) presented another approach to the SOD leading to maximum reliability using linear programming (see e.g. Bazaraa 1974; Smith et al. 1983). Amiri-Seemkooei (2004) presented a method for the SOD. Doma (2014) developed another method for the SOD in a monitoring network and compared it with Kuang's (1996) method and concluded the both of them can meet the precision criterion of the strain parameters.

By using the method of Kuang (1996), one can obtain optimal weights and configuration of the network in one step by different optimisation algorithms and OFs. In fact, the approach proposed by him is a combination of FOD and SOD. In this method the best configuration and observation precisions are determined simultaneously in an optimal way. Amiri-Seemkooei (2001a,b) considered the analytical approach to FOD, SOD and also their combinations in robustness points of view. This optimal design can be carried out using different criteria as an OF. Alzubaidy et al. (2012) discussed the problem of the FOD and SOD in a microgeodetic network. Amiri-Seemkooei et al. (2012) presented some basic concepts related to the optimisation and design of geodetic networks. Mehrabi and Voosoghi (2014) optimised the observational plan of a local GPS network concerning the precision criterion. If just one criterion exists in the OF, it is called single-objective optimisation model (SOOM); if two criteria exist, it is a bi-objective optimisation model (BOOM) (Mehrabi 2002). A simple comparison between different SOOMs has been carried out in Eshagh and Kiamehr (2007). This comparison shows that reliability is a better criterion than the other criteria in SOOMs. The capability of BOOM versus SOOM was presented in Eshagh (2005). Bagherbandi et al. (2009) compared SOOM and MOOM in a simulated geodetic network and concluded the superiority of MOOM with respect to SOOM.

In this study, our goal is to find out which one of the precision and reliability constraints is more suitable for the network optimisation by BOOM of reliability and precision. It is clear 
that considering both of them leads to better results, but the question is if we use just one or none of them what happens to the optimised network. A simple geodetic network is designed and optimised for this purpose and the results of the optimisation based on these constraints are presented.

\section{Bi-objective optimisation model}

Since SOOMs are restricted to the constraints' inconsistencies, thus if the criterion matrix of the station coordinates and the criterion vector of the reliability are not defined properly the constraints will be inconsistent and no solution exists. BOOM is a method to solve this restriction (Kuang 1996; Amiri-Seemkooei 1998; Mehrabi 2002). Here, we define the $\mathrm{BOOM}$ of precision and reliability in the following $\mathrm{OF}$, which is suitable for two-dimensional networks:

$$
\left[\frac{\|\mathbf{H w}-\mathbf{u}\|}{\left\|\operatorname{vec}\left(\mathbf{C}_{S}\right)\right\|}+\frac{\left\|\mathbf{R}_{11} \mathbf{w}-\left(\mathbf{r}_{\min }-\mathbf{r}_{00}\right)\right\|}{\left\|\mathbf{r}_{\min }\right\|}\right] \rightarrow \min
$$

subject to

$$
\begin{aligned}
& {\left[\mathbf{D}^{\mathrm{T}} \mathbf{0}\right] \mathbf{w}=\mathbf{0}} \\
& \mathbf{A}_{00} \mathbf{w} \leq \mathbf{b}_{00}
\end{aligned}
$$

where $\mathbf{w}=\left[\Delta x_{1} \Delta y_{1} \ldots \Delta x_{m} \Delta y_{m} \Delta p_{1} \ldots \Delta p_{n}\right]$ is the vector of the improvements, shown by $\Delta$, to the station coordinates $x_{i}, y_{i}, \quad i=1, \ldots, m . m$ represents the number of net points and to the observational weights $p_{j}, j=1,2, \ldots n$ where $n$ stands for number of observations. The $\operatorname{vec}(\bullet)$ operator converts a matrix to a single column vector by stacking the columns of a matrix one below another, $\|\bullet\|$ stands for the $L_{2}$-norm, $\mathbf{r}_{\min }$ is the acceptable level of reliability for each observable. $\mathbf{D}$ is called the datum matrix and the superscript $(\bullet)^{\mathrm{T}}$ shows the transpose operator. $\mathbf{H}$ is the coefficients matrix derived by linearising the precision criterion matrix $\mathbf{C}_{s}$ and $\mathbf{u}$ is the vector of differences between $\mathbf{C}_{s}$ and the initial variancecovariance matrix of the unknowns $\mathbf{C}_{x}^{0}$. The criterion matrix is an ideal variance-covariance matrix, which is transferred to the datum of the network by S-Transformation as:

$$
\mathbf{C}_{s}=\mathbf{S C S}^{\mathrm{T}}
$$

where $\mathbf{S}=\mathbf{I}_{m}-\mathbf{D}^{\mathrm{T}}\left(\mathbf{D D}^{\mathrm{T}}\right)^{-1} \mathbf{D}$ and $\mathbf{C}$ is the initial criterion matrix. $\mathbf{I}_{m}$ represents the identity matrix of dimension $m$. Note that the OF, presented in Eq. (1), contains two terms and the first one expresses the precision criterion and the second one, the reliability.

$\mathbf{H}$ and $\mathbf{u}$ in Eq. (1) have the following structures:

$$
\begin{aligned}
& \mathbf{H}=\left[\operatorname{vec}\left(\frac{\partial \mathbf{C}_{x}}{\partial x_{1}}-\frac{\partial \mathbf{C}_{s}}{\partial x_{1}}\right) \quad \operatorname{vec}\left(\frac{\partial \mathbf{C}_{x}}{\partial y_{1}}-\frac{\partial \mathbf{C}_{s}}{\partial y_{1}}\right) \ldots\right. \\
& \operatorname{vec}\left(\frac{\partial \mathbf{C}_{x}}{\partial x_{m}}-\frac{\partial \mathbf{C}_{s}}{\partial x_{m}}\right) \quad \operatorname{vec}\left(\frac{\partial \mathbf{C}_{x}}{\partial y_{m}}-\frac{\partial \mathbf{C}_{s}}{\partial y_{m}}\right) \\
& \left.\operatorname{vec}\left(\frac{\partial \mathbf{C}_{x}}{\partial p_{1}}\right) \quad \cdots \quad \operatorname{vec}\left(\frac{\partial \mathbf{C}_{x}}{\partial p_{n}}\right)\right] \\
& \mathbf{u}=\operatorname{vec}\left(\mathbf{C}_{s}\right)-\operatorname{vec}\left(\mathbf{C}_{x}^{0}\right)
\end{aligned}
$$

where $\mathbf{C}_{x}$ is the variance-covariance matrix of the unknowns.

In Eq. (1), $\mathbf{R}_{11}$ has the following matrix form:

$$
\mathbf{R}_{11}=\left(\mathbf{I}_{n} \Theta \mathbf{I}_{n}\right)^{\mathrm{T}} \mathbf{R}_{1}
$$


where $\mathbf{I}_{n}$ is the identity matrix with dimension of $n$. $\Theta$ represents the Khatri-Rao (Khatri and Rao 1968) product and $\mathbf{R}_{1}$ is defined by:

$$
\begin{array}{r}
\mathbf{R}_{1}=\left[\operatorname{vec}\left(\frac{\partial \mathbf{R}}{\partial x_{1}}\right) \quad \operatorname{vec}\left(\frac{\partial \mathbf{R}}{\partial y_{1}}\right) \quad \ldots\right. \\
\operatorname{vec}\left(\frac{\partial \mathbf{R}}{\partial x_{m}}\right) \quad \operatorname{vec}\left(\frac{\partial \mathbf{R}}{\partial y_{m}}\right) \quad \ldots \\
\left.\operatorname{vec}\left(\frac{\partial \mathbf{R}}{\partial p_{1}}\right) \quad \cdots \quad \operatorname{vec}\left(\frac{\partial \mathbf{R}}{\partial p_{n}}\right)\right] .
\end{array}
$$

In Eq. (8), $\mathbf{R}$ stands for the reliability matrix of the network, which can be obtained from the residual vector $\hat{\mathbf{v}}$ (Kuang 1996, p. 122):

$$
\hat{\mathbf{v}}=\hat{\mathbf{L}}-\mathbf{L}=\left[\mathbf{A}\left(\mathbf{A}^{\mathrm{T}} \mathbf{P A}+\mathbf{D} \mathbf{D}^{\mathrm{T}}\right)^{-1} \mathbf{A}^{\mathrm{T}} \mathbf{P}-\mathbf{I}_{n}\right] \mathbf{L}=-\mathbf{R} \mathbf{L}
$$

where $\mathbf{L}$ is the observation vector and $\hat{\mathbf{L}}$ is its estimated one after adjustment process. According to Eq. (9), $\mathbf{R}$ can be written as follows:

$$
\mathbf{R}=\mathbf{I}_{n}-\mathbf{A}\left(\mathbf{A}^{\mathrm{T}} \mathbf{P} \mathbf{A}+\mathbf{D} \mathbf{D}^{\mathrm{T}}\right)^{-1} \mathbf{A}^{\mathrm{T}} \mathbf{P}
$$

where $\mathbf{A}$ and $\mathbf{P}$ are the design and weight matrices, which are used in the least-squares adjustment of the network, respectively. In fact, $\mathbf{R}_{1}$ is the matrix of the derivatives of $\mathbf{R}$ with respect to the elements of vector $\mathbf{w}$; and $\mathbf{R}_{11}$ contains the diagonal elements of $\mathbf{R}_{1}$. The role of $\left(\mathbf{I}_{n} \Theta \mathbf{I}_{n}\right)^{\mathrm{T}}$ in Eq. (7) is to consider the diagonal elements of $\mathbf{R}_{1}$ in the reliability constraint.

In order to have some ideas about the reliability of the existing network, we compute the reliability of all observables and organise the vector $\mathbf{r}^{0}$, which $\mathbf{r}^{0}=\operatorname{vec}\left(\mathbf{R}^{0}\right)$, where $\mathbf{R}^{0}$ is the initial value of $\mathbf{R}$. $\mathbf{r}_{00}$ or the diagonal elements of $\mathbf{R}^{0}$ can be selected by:

$$
\mathbf{r}_{00}=\left(\mathbf{I}_{n} \Theta \mathbf{I}_{n}\right)^{\mathrm{T}} \mathbf{r}^{0}
$$

and $\mathbf{R}^{0}$ has the following structure:

$$
\mathbf{R}^{0}=\left[\mathbf{I}_{n}-\mathbf{A}\left(\mathbf{A}^{\mathrm{T}} \mathbf{P A}+\mathbf{D} \mathbf{D}^{\mathrm{T}}\right)^{-1} \mathbf{A}^{\mathrm{T}} \mathbf{P}\right]_{\mathbf{w}^{0}}
$$

where $\mathbf{w}^{0}$ is the initial value of $\mathbf{w}$.

In Eq. (3), $\mathbf{A}_{00}$ is the coefficient matrix of the inequality constraints and $\mathbf{b}_{00}$ is the constant vector of them. One can see their structures below:

$$
\mathbf{A}_{00}=\left[\begin{array}{c}
\mathbf{I}_{2 m+n} \\
-\mathbf{I}_{2 m+n}
\end{array}\right]
$$

and

$$
\begin{aligned}
\mathbf{b}_{00}= & {\left[\begin{array}{lllllllll}
a_{21} & b_{21} & \cdots & a_{2 m} & b_{2 m} & \Delta p_{1} & \cdots & \Delta p_{n} \\
& -a_{11} & -b_{11} & \cdots & -a_{1 m} & -b_{1 m} & p_{1}^{0} & p_{n}^{0}
\end{array}\right]^{\mathrm{T}} }
\end{aligned}
$$

where $\left[a_{1 i}, a_{2 i}\right]$ and $\left[b_{1 i}, b_{2 i}\right]$ are the boundary values of the unknown coordinate changes $\Delta x_{i}, \Delta y_{i}, i=1, \cdots, m$ and $\Delta p_{j}, j=1, \cdots, n$ are the improvements to the initial weights $p_{j}^{0}$.

The solution of the above problem leads to an ideal point representing both of the precision and reliability requirements for the network. In fact, the OF, presented in Eq. (1), minimises the differences amongst these criteria and subjects them to the physical constraints presented 
in Eqs. (13) and (14) in a least-squares sense. By applying $L_{2}$-norm to the model (1), the $\mathrm{BOOM}$ is converted to the following quadratic programming model:

$$
\left(\mathbf{w}^{\mathrm{T}} \mathbf{H}_{0}^{\mathrm{T}} \mathbf{H}_{0} \mathbf{w}-2 \mathbf{u}_{0}^{\mathrm{T}} \mathbf{H}_{0} \mathbf{w}+\mathbf{u}_{0}^{\mathrm{T}} \mathbf{u}_{0}\right) \rightarrow \min
$$

where

$$
\mathbf{H}_{0}=\left[\frac{\mathbf{H}}{\sqrt{\mathbf{u}^{\mathrm{T}} \mathbf{u}}} \frac{\mathbf{R}_{11}}{\sqrt{\mathbf{r}_{\min }^{\mathrm{T}} \mathbf{r}_{\min }}}\right] \text { and } \quad \mathbf{u}_{0}=\left[\frac{\mathbf{u}}{\sqrt{\left[\operatorname{vec}\left(\mathbf{C}_{s}\right)\right]^{\mathrm{T}}\left[\operatorname{vec}\left(\mathbf{C}_{s}\right)\right]}} \frac{\mathbf{r}_{\min }-\mathbf{r}_{00}}{\sqrt{\mathbf{r}_{\min }^{\mathrm{T}} \mathbf{r}_{\min }}}\right]^{\mathrm{T}}
$$

subject to

$$
\begin{aligned}
{\left[\mathbf{D}^{\mathrm{T}} \mathbf{0}\right] \mathbf{w} } & =\mathbf{0} \\
\mathbf{A}_{22} \mathbf{w} & \leq \mathbf{b}_{22}
\end{aligned}
$$

Note that the inequality constraints in Eq. (18) can include the precision and/or reliability controlling constraints. The structures of $\mathbf{A}_{22}$ and $\mathbf{b}_{22}$ are:

$$
\begin{aligned}
& \mathbf{A}_{22}=\left[\begin{array}{l}
\mathbf{A}_{00} \\
\mathbf{A}_{11}
\end{array}\right] \text { and } \mathbf{A}_{11}=\left[\begin{array}{c}
\mathbf{H}_{1} \\
-\mathbf{R}_{11}
\end{array}\right] \\
& \mathbf{b}_{22}=\left[\begin{array}{l}
\mathbf{b}_{00} \\
\mathbf{b}_{11}
\end{array}\right] \text { and } \quad \mathbf{b}_{11}=\left[\begin{array}{c}
\mathbf{u}_{1} \\
\mathbf{r}_{00}-\mathbf{r}_{\min }
\end{array}\right]
\end{aligned}
$$

where

$$
\begin{aligned}
\mathbf{H}_{1} & =\left(\mathbf{I}_{2 m} \Theta \mathbf{I}_{2 m}\right)^{\mathrm{T}} \mathbf{H} \\
\mathbf{u}_{1} & =\left(\mathbf{I}_{2 m} \Theta \mathbf{I}_{2 m}\right)^{\mathrm{T}} \mathbf{u} .
\end{aligned}
$$

It can be seen in Eq. (19) that $\mathbf{A}_{11}$ and $\mathbf{b}_{11}$ have two sub-matrices, which represent the precision by $\mathbf{H}_{1}, \mathbf{u}_{1}$ and the reliability by $-\mathbf{R}_{11}, \mathbf{r}_{00}-\mathbf{r}_{\min }$. Based on the requirements of the project, one can use either one or both of them. In order to solve the quadratic programming problem presented in Eq. (15), one has to transfer it to a linear programming problem which is called the linear complementary problem, which its solution gives the optimal values for the quadratic programming; for more details see e.g. Lemke (1962) or Koch (1985).

\section{Numerical studies}

In order to test the effect of the precision and reliability constraints on optimisation, a simple trilateration geodetic network is simulated. The network is horizontal and consists of seven points and it is assumed that all possible distances amongst the net points are measurable. Here, ZOD is done by selecting Point 4 as a fixed point against the network translation, and the direction from this point to Point 1 is considered as the fixed direction to preserve the network against the rotation. These conditions have been found based on the S-transformation (Teunissen 1985) and the precision criterion.

In order to optimise this network by the BOOM of reliability and precision, we assume that the errors of the net points after optimisation should be all equal to $2 \mathrm{~mm}$ and the reliabilities of the observations are to be larger than 0.4 . Also, we assume that to get an optimised configuration we can relocate the net points by $\pm 2 \mathrm{~m}$. In the first step of our study, we optimise the network using BOOM but without any constraint. The optimised network and the size of error ellipses, which are plotted 5,000 times larger, are shown in Fig. 1a. The size of the ellipses becomes smaller as expected. In the second step, BOOM is subjected to 
(a)

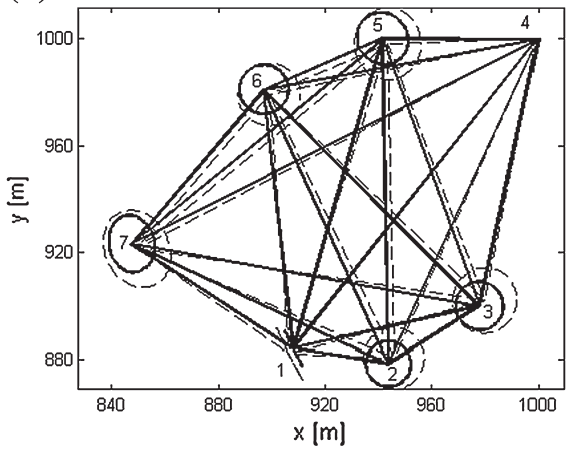

(c)

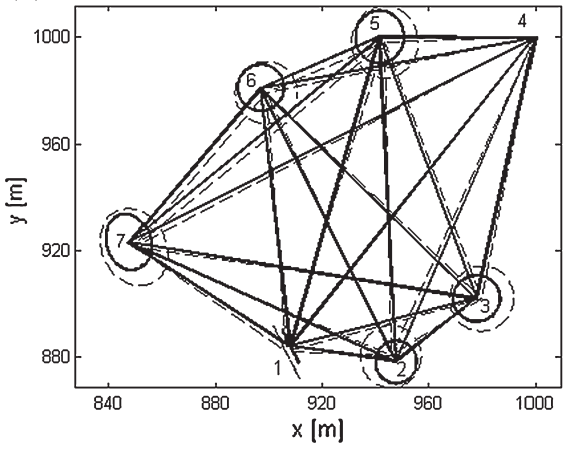

(b)

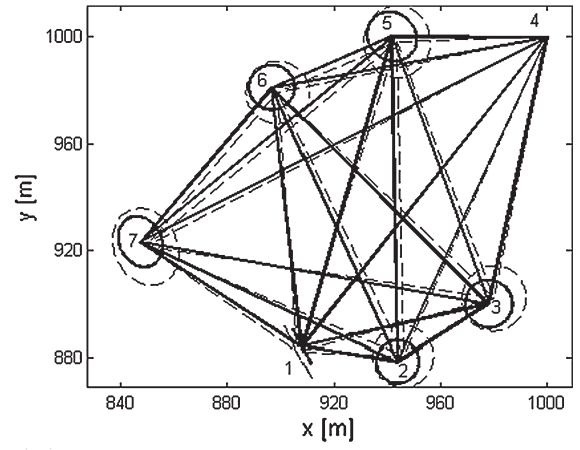

(d)

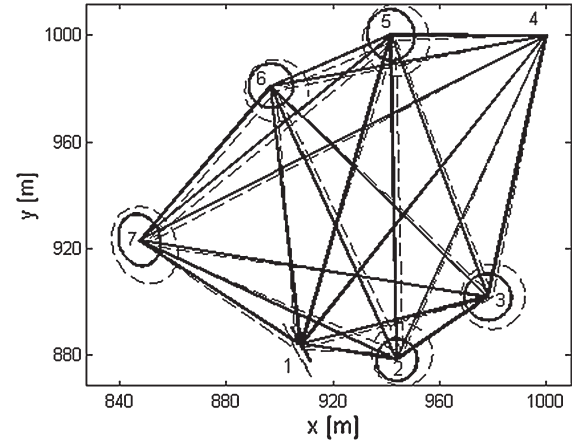

Fig. 1 Optimised network and error ellipses by BOOM subjected to a desired accuracy of $2 \mathrm{~mm}$ for different types of constraints: a unconstraint, $\mathbf{b}$ precision, $\mathbf{c}$ reliability and $\mathbf{d}$ precision and reliability. Network before optimisation and initial error ellipses for net points are illustrated by dashed lines

the precision constraint and the network is optimised and the result is plotted in Fig. 1b. As can be seen, the error ellipses are slightly smaller than those obtained in the case of using BOOM without constraint, especially for Point 7 , which is the furthest point from the fixed point of the network or Point 4. When BOOM is subjected only to the reliability constraint, nothing especial happens in the optimised network except the error ellipse of Point 6 , which is slightly smaller than the one obtained in the unconstrained optimisation. This means that the reliability constraint cannot significantly help to change the quality of the coordinate of the net points, this can be seen in Fig. 1c, which is almost the same with Fig. 1a except for Point 2. If BOOM is subjected to both of the precision and reliability constraints the optimised network will be more or less the same with the case of BOOM with the precision constraint. Similarity of Fig. 1d, b shows the insignificant influence of the reliability constraint in the optimisation process in the precision point of view.

In the previous case, we observed that no observable was removed from the optimised network. Now, we perform another test by considering a larger error of $3 \mathrm{~mm}$ for the error of net points. In fact, we would like to have a less accurate network than the existing one. Figure 2a shows the optimised network by unconstrained BOOM, and as we expected the error ellipses become larger now, and 6 observables can be removed to reach to an accuracy level of $3 \mathrm{~mm}$. In the case of subjecting BOOM to the precision constraint, 4 observables can be removed, see Fig. 2b, which means that the unconstrained BOOM already had some 
(a)

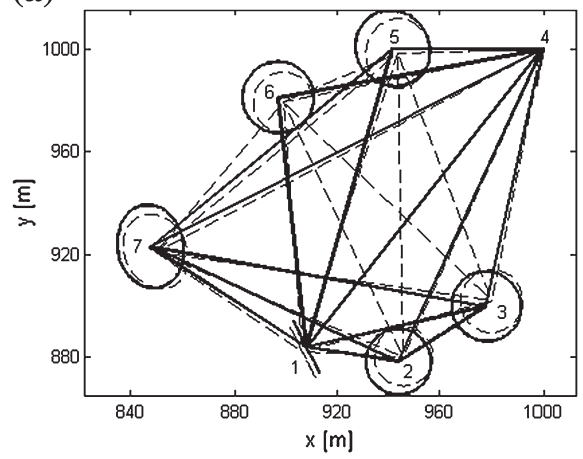

(c)

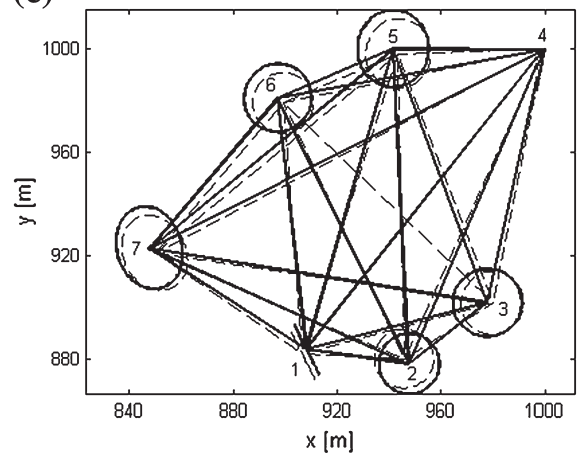

(b)

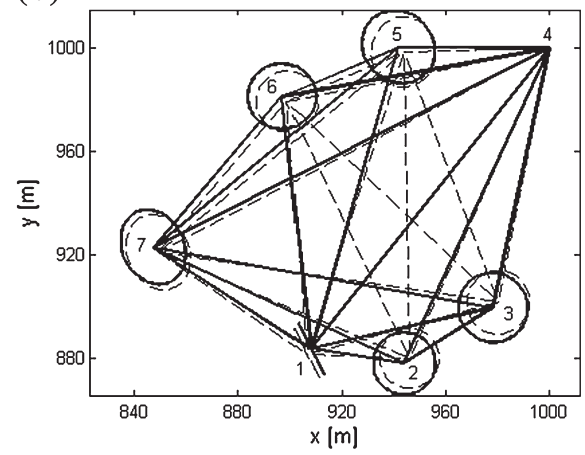

(d)

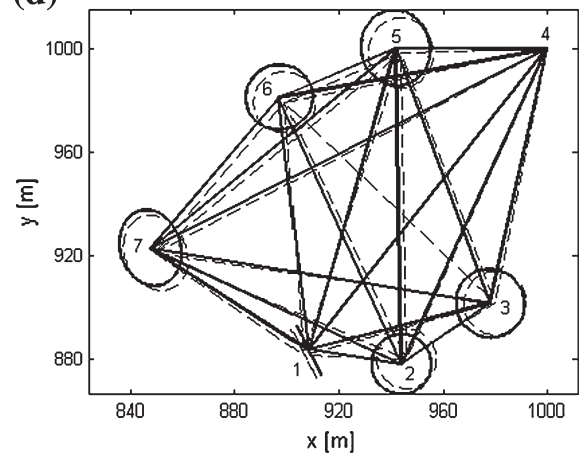

Fig. 2 Optimised network and error ellipses by BOOM subjected to a desired accuracy of $3 \mathrm{~mm}$ for different types of constraints: a unconstraint, $\mathbf{b}$ precision, $\mathbf{c}$ reliability and $\mathbf{d}$ precision and reliability. Network before optimisation and initial error ellipses for net points are illustrated by dashed lines

observables with larger errors than $3 \mathrm{~mm}$. Figure $2 \mathrm{c}$ shows the configuration of the network after optimisation with the reliability constraint and Fig. $2 d$ shows the network optimised based on BOOM subjected to the reliability and precision constraints. The optimised network is very similar to the one obtained by using only the precision constraint.

Table 1 presents the errors of the net points before and after optimisation based on BOOM in both cases of considering desired errors of 2 and $3 \mathrm{~mm}$. The errors of the points before the optimisation is presented in the first column of the table and as it shows the largest error is related to Point 7, which is the furthest point from Point 4. The least error belongs to Point 1 because the direction of Point 4 to this point is kept fixed and this point has less freedom to change during the optimisation process. Except Point 1, the errors of the rest of the points are larger than $2 \mathrm{~mm}$ and, here, we want to homogenise the network by optimising it in such a way that the errors of all points become $2 \mathrm{~mm}$. The column showed by U means optimised by the unconstrained BOOM, P means constrained to the precision, R to reliability and PR both of them. In the case of using the unconstrained BOOM, the errors of all points become smaller than $2 \mathrm{~mm}$ expect that of Point 7, which is slightly larger. When BOOM is constrained to the reliability model the result is the same with the case of unconstrained BOOM and in the case of using constrained BOOM to precision, having errors of all points less than $2 \mathrm{~mm}$ is guaranteed. This will be very similar to the case of using both of the precision and reliability constraints. Similar issues can be seen when the desired error is $3 \mathrm{~mm}$ for all points and 
Table 1 Errors of net points before and after optimisation by BOOM, Unconstrained (U), Precision (P), Reliability (R) and Precision and Reliability (PR)

\begin{tabular}{|c|c|c|c|c|c|c|c|c|c|}
\hline & \multirow[t]{2}{*}{ Before opt. } & \multicolumn{4}{|c|}{ Desired accuracy: $2 \mathrm{~mm}$} & \multicolumn{4}{|c|}{ Desired accuracy: $3 \mathrm{~mm}$} \\
\hline & & $\mathrm{U}$ & $\mathrm{P}$ & $\mathrm{R}$ & PR & $\mathrm{U}$ & $\mathrm{P}$ & $\mathrm{R}$ & PR \\
\hline$\sigma_{x_{1}}$ & 1.3 & 0.9 & 0.9 & 0.9 & 0.9 & 1.4 & 1.4 & 1.4 & 1.4 \\
\hline$\sigma_{y_{1}}$ & 1.7 & 1.2 & 1.2 & 1.2 & 1.1 & 1.8 & 1.7 & 1.7 & 1.7 \\
\hline$\sigma_{x_{2}}$ & 2.3 & 1.7 & 1.6 & 1.6 & 1.5 & 2.5 & 2.4 & 2.3 & 2.3 \\
\hline$\sigma_{y_{2}}$ & 2.3 & 1.7 & 1.6 & 1.6 & 1.6 & 2.5 & 2.4 & 2.3 & 2.3 \\
\hline$\sigma_{x_{3}}$ & 2.2 & 1.8 & 1.8 & 1.6 & 1.6 & 2.7 & 2.6 & 2.4 & 2.4 \\
\hline$\sigma_{y_{3}}$ & 2.5 & 1.7 & 1.7 & 1.8 & 1.8 & 2.6 & 2.6 & 2.6 & 2.7 \\
\hline$\sigma_{x_{5}}$ & 2.4 & 1.8 & 1.7 & 1.8 & 1.7 & 2.7 & 2.5 & 2.7 & 2.6 \\
\hline$\sigma_{y_{5}}$ & 2.4 & 1.9 & 1.8 & 1.8 & 1.8 & 2.9 & 2.8 & 2.7 & 2.7 \\
\hline$\sigma_{x_{6}}$ & 2.3 & 1.8 & 1.6 & 1.6 & 1.6 & 2.6 & 2.3 & 2.4 & 2.3 \\
\hline$\sigma_{y_{6}}$ & 2.3 & 1.9 & 1.7 & 1.8 & 1.7 & 2.8 & 2.6 & 2.7 & 2.5 \\
\hline$\sigma_{x_{7}}$ & 1.9 & 1.3 & 1.3 & 1.3 & 1.2 & 2.0 & 1.9 & 1.9 & 1.9 \\
\hline$\sigma_{y_{7}}$ & 3.0 & 2.2 & 2.0 & 2.2 & 2.0 & 3.3 & 3.0 & 3.2 & 3.0 \\
\hline
\end{tabular}

optimisation using BOOM shows that the errors become larger. In the cases where BOOM is subjected to the precision constraint the errors of the points are smaller than $3 \mathrm{~mm}$.

Table 2 shows the values of the reliabilities and weights of observables after optimisation by BOOM with different types of constraints. The second column from the left hand side of the table shows the initial reliability of the observables before the optimisation process. If we assume that 0.4 is the acceptable reliability level, we can conclude that the non-optimised network is already reliable and can resist against the gross errors. In the case of optimising it, considering the precision of $2 \mathrm{~mm}$, without any constraint three observables of L17, L34 and L45 will have smaller reliability than 0.4. If we look at Fig. 2a we observe that these observables are in the marginal areas of the network and it is quite normal to see low reliabilities for them; but they have a common value of 0.3 , which may not be very significant in practice. The column $\mathrm{R}$ shows that by considering the reliability constraint the reliability numbers of all observables will be larger than 0.4. If the optimisation is constrained only to precision, L34 will have a reliability of 0.3 . This shows that the precision constraint can somehow improve the reliability of the network as well. Considering both of the constraints leads to a network with homogenous error distribution of all points with less than $2 \mathrm{~mm}$ and a reliability of higher than 0.4 for all observables. Another issue is that when the precision criterion is $2 \mathrm{~mm}$ none of the observables is removed during the optimisation process. If this network is optimised by a SOOM with these two constraints the contradiction between the constraints may happen and the solution may not be feasible. This is the issue, which has been studied by $\mathrm{Xu}$ (1989) and he mentioned that the use of BOOM is one way to avoid such a contradiction. The reason is that in SOOM the optimisation is done by concentrating the problem on either precision or reliability constraint, but in BOOM, both of these criteria are considered in one single OF. In fact, BOOM is an OF having both properties of minimising the error and maximising the reliability whilst SOOM can have only one of them.

In the case of considering an error of $3 \mathrm{~mm}$ for all points, we will observe that, again, L17, L34 and L45 will get a reliability of 0.3 and by considering the reliability constraint all reliabilities become higher than 0.4 in the network. When BOOM is subjected to no constraint, 


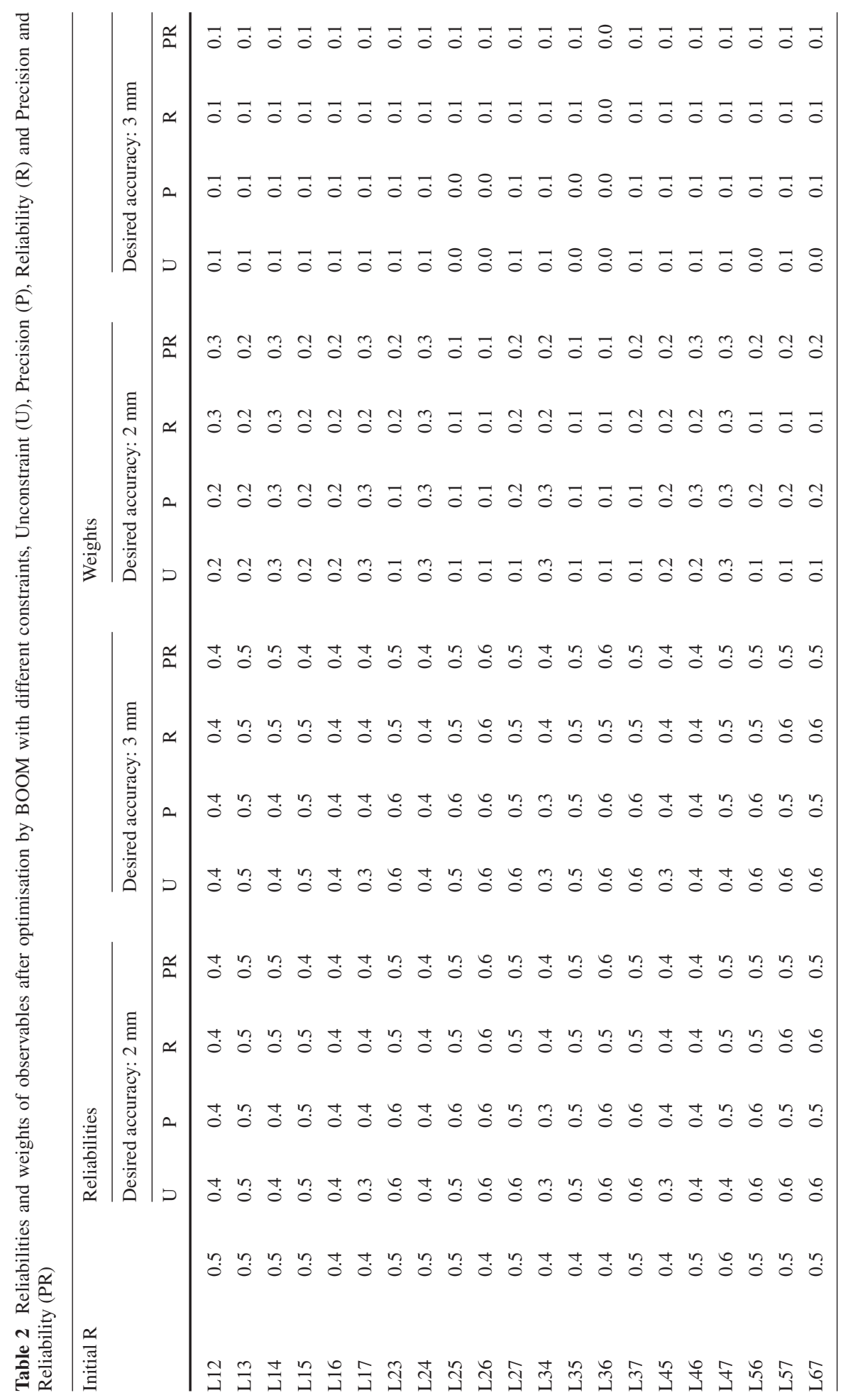


6 observable of L25, L26, L35, L36, L56 and L67 can be removed from the observation plan. By looking at Fig. 2a we see that these lengths are inside the network improving its geometric strength. Removal of these lengths means that based on the requested accuracy, $3 \mathrm{~mm}$, and such a geometric strength of the network caused by the inner observations is not required. In the case of constraining BOOM to precision, four observables of L25, L26, L35 and L36 are insignificant and in the case of considering the reliability constraint only L36. This means that the reliability constraint tries to keep the number of observables large so that they can control each other. In other words, in order to increase the reliability of one observable the rest of reliabilities should vary in such a way that their sum remains equal to the redundancy of the network. It can be seen in the table that the observables with the least reliabilities are not necessarily the ones, which should be deleted from the network after optimisation. For example, in the case of considering $3 \mathrm{~mm}$ error for the points and in the unconstrained case, L17, L34 and L45 have the least reliability but of L25, L26, L35, L36, L56 and L67 are removable, which are totally different. The reliabilities of these removable observables are all higher than 0.4 , which mean that for the precision of $3 \mathrm{~mm}$ the network does not need these high reliable lengths.

\section{Conclusions}

In this simulation study, we observed that our BOOM of precision and reliability can optimise the network properly even without controlling constraints. Although the errors of some net points and the reliabilities of some observables do not match the required accuracy and reliability, the differences are not very large and considerable in practice. In the case of expecting a less accurate network comparing to the existing one, those observations which are placed in the middle of the network and have the main role in the network geometrical strength are removed. This shows that a simple network with a lower redundancy is enough for reaching to that accuracy. Here, according to this simple numerical study we conclude that the use of unconstraint BOOM is more economical in practical considerations as more observables are deleted from the plan and the accuracy and reliability of the network almost meet the network requirements. Furthermore, the less reliable observables are those, which are placed in the marginal areas of the network and they are not those, which should be deleted from the observation plan.

Acknowledgments The authors would like to thank FORMAS for granting Project DNR-245-2012-356 and Professor Lars E. Sjöberg at Royal Institute of Technology (KTH) in Sweden.

\section{References}

Alzubaidy RZ, Mahdi HA, Hanooka HS (2012) Optimized zero and first order design of micro geodetic networks. J Eng 18(12):1344-1364

Amiri-Seemkooei A (1998) Analytical methods in optimization and design of geodetic networks. K. N. Toosi University of Technology, Surveying Engineering, Tehran

Amiri-Seemkooei A (2001a) Comparison of reliability and geometrical strength criteria in geodetic networks. J Geod 75(4):227-233

Amiri-Seemkooei A (2001b) Strategy for designing geodetic network with high reliability and geometrical strength criteria. J Surv Eng 127(3):104-117

Amiri-Seemkooei A (2004) A new method for second-order design of geodetic networks: aiming at highreliability. Surv Rev 37(293):552-560

Amiri-Seemkooei A (2008) Analytical first-order design of geodetic networks. Iran J Eng Sci 1(1):1-12 
Amiri-Seemkooei A, Asgari J, Zangeneh-Nejad F, Zaminpardaz S (2012) Basic concepts of optimization and design of geodetic networks. J Surv Eng 138(4):172-183

Bagherbandi Mohammad, Eshagh Mehdi, Sjöberg Lars Erik (2009) Multi-objective versus single-objective models in geodetic network optimization. Nordic J Surv Real Estate Res 6(1):7-20

Bazaraa MS (1974) Quadratic set covering problem. Georgia Institute of Technology, Atlanta

Bazaraa MS, Shetty CM (1979) Nonlinear programming. Wiley, New York

Berne JL, Baselga S (2004) First-order design of geodetic networks using the simulated annealing method. J Geod 78:47-54

Blewitt G (2000) Geodetic network optimization for geophysical parameters. Geophys Res Lett 22(27): 2615-3618

Doma MIA (2013) Particle swarm optimization in comparison with classical optimization for GPS network design. J Geod Sci 3(4):250-257

Doma MIA (2014) A comparison of two different measures of precision into geodetic deformation monitoring networks. Arab J Sci Eng 39:695-704

Eshagh M (2005) Optimization and design of geodetic networks. Islamic Azad University, Shahr-e-Rey branch, Tehran

Eshagh M, Kiamehr R (2007) A strategy for optimum designing of the geodetic networks from the cost, reliability and precision views. Acta Geodaetica et Geophysica Hungarica 42(3):297-308

Gerasimenko MD, Shestakov NV, Kato T (2000) On optimal geodetic network design for fault-mechanics studies. Earth Planets Space 52:985-987

Grafarend EW (1975) Second order design of geodetic nets. Zeitschrift für Vermessungswesen 100:158-168

Khatri CG, Rao CR (1968) Solutions to some functional equations and their applications to characterization of probability distributions. Sankhya 30:167-180

Koch KR (1982) Optimization of the configuration of geodetic networks. Deutsche Geodaetische Kommission 3(258):82-89

Koch Karl Rudolf (1985) First order design: optimization of the configuration of a network by introducing small position changes. In: Grafarend EW, Sanso F (eds) Optimization and design of geodetic networks. Springer, Berlin, pp 56-73

Kuang S (1991) Optimization and design of deformation monitoring schemes. PhD thesis, Department of Surveying Engineering, University of New Brunswick, Fredericton, Canada

Kuang S (1993) Second order design: shooting for maximum reliability. J Surv Eng 119(3):102-110

Kuang S (1996) Geodetic network analysis and optimal design: concepts and applications. Ann Arbor Press, Chelsea

Lemke CE (1962) A method of solution for quadratic programs. Manag Sci 8(4):442-453

Mehrabi H (2002) Fully-analytical approach to bi-objective optimization and design of geodetic networks. Master Thesis, Geodesy and Geomatics, K. N. Toosi Technical University, Tehran

Mehrabi H, Voosoghi B (2014) Analytical method in selecting optimal observational plan of local GPS networks. J Geod Sci 4(1):87-97

Peiliang X (1989) On robust estimation with correlated observations. Bull géod 63(3):237-252

Schmitt G (1980) Second order design of free distance networks considering different types of criterion matrices. Bull Geod 54:531-543

Schmitt G (1985) Second order design. In: Grafarend EW, Sanso F (eds) Optimization and design of geodetic networks. Springer, Berlin, pp 74-121

Schmitt G (1985) Third order design. In: Grafarend EW, Sanso F (eds) Optimization and design of geodetic networks. Springer, Berlin, pp 122-131

Smith Alan A, Hinton Ernest, Lewis Roland W (1983) Civil engineering systems analysis and design. Wiley, New York

Teunissen P (1985) Zero order design: generalized inverses, adjustment, the datum problem and Stransformations. In: Optimization and design of geodetic networks, Springer, Berlin, Heidelberg, pp $11-55$

Yetki M, Cevat I, Yigit CO (2008) Optimal Design of Deformation Monitoring Networks using PSO Algorithm. In: 13th FIG symposium on deformation measurement and analysis, 4th IAG symposium on geodesy and geotechnical and structural engineering, 12-15 May 2008 